(6 January, pp 7 and 10), we feel that some critics may have rushed to defend the forceps without reading our: papers carefully. Of course, many different intrapartum events might generate neonatal morbidity in association with the use of Kielland's forceps. However, we clearly demonstrated an excess of babies with abnormal neurological behaviour even in the absence of preceding fetal asphyxia. Furthermore, we looked for an adverse influence of mechanical factors operating during labour, and, within the limits of a retrospective study, we did not find it.

We agree that a prospective controlled trial of different ways of managing malposition is necessary to assess accurately the neonatal morbidity caused by Kielland's forceps. Indeed, we stated that long-term follow-up studies of babies were needed to assess properly the role of caesarean section for malposition. Pending such investigations one should not dismiss any retrospective study that draws attention to neonatal problems associated with current obstetric practice-a sentiment that incidentally is supported by the tone of your leading article on the subject (10 February, p 362).

Neonatal Medical Unit, St Mary's Hospital,

Manchester

Department of Obstetrics and

MaLCOLM L CHISWICK

Gynaecology

Wythenshawe Hospital,

${ }^{*}{ }^{*}$ This correspondence is now closed.-ED, $B M 7$.

\section{“Doctors' orders"}

SIR,-We should like to raise two points which arise from the interesting article by Dr D A Ellis and others (17 February, p 456).

Communication is a two-way process and, while written information may have improved the patient's reception and therefore understanding and recall of advice, the exercise of writing down the advice may have meant that it was given in a more lucid and concise way by the doctor. Whatever the mechanism, improving the communication of 'doctors' orders" to patients is an important step forward.

During investigations of the circumstances leading up to the deaths of infants, both at home and shortly after admission to hospital, we have been disturbed by parents' lack of understanding of advice given by genera practitioners during consultations in the children's terminal illness. Particular difficulties have arisen not so much with adherence to drug dose schedules (as these are usually written on bottle labels by the pharmacist) as with how the parents should recognise deterioration in the children's illness, how long medication can be expected to take to work, what constitutes deterioration, and what to do should deterioration in the condition occur. Clear instructions should be left with the parents that they should not wait for days for medication to be effective, and that deterioration in the child's illness-as marked by increasing severity or increasing numbers of symptoms-should be an indication for recalling the general practitioner.

We feel that written instructions would be as valuable in the primary care situation as in hospital. They would, in many cases, mean that "doctors' orders" were more concise and adequate in themselves, thus helping parents' understanding of them, and also enabling them to check instructions at a later time. As a result of this, infants would receive earlier medical reappraisal of illness when necessary, with possible lowering of morbidity and mortality.

J R OAKLEY

Children's Hospital, P M MCWEENY Sheffield

Thrombocytopenia and splenic infarction

SIR,-Dr P T Wilmshurst and others (10 February, p 381) have reported thrombocytopenia in a patient with renal carcinoma which they think may have been related to splenic infarction. This is an interesting suggestion, as important splenic infarction is usually associated with a raised platelet count as a result of hyposplenism. ${ }^{1}$ In the absence of disseminated intravascular coagulation, thrombocythaemia is also the more common platelet abnormality associated with carcinomas. ${ }^{2}$

As the patient had a palpable spleen, it is important that the possibility of hypersplenism should be considered. It would be helpful to know: (a) the weight of the spleen at necropsy and $(b)$ should splenic infarction have occurred at a late stage in the patient's illness, whether terminally there was a palpable spleen and thrombocytopenia. The hypersplenism could be either idiopathic or secondary to splenic congestion.

I would be happier to accept the suggestion of Wilmhurst et al that the thrombocytopenia was due to splenic infarction if hypersplenism could be excluded, and if "the diffuse intravascular coagulation screen" ruled out the possibility of chronic consumption coagulopathy-the latter being very likely in the presence of a mass of necrotic tissue."

D O HO-YEN

Haematology Department,

Ninewells Hospital,

undee

Crosby, W H, Annual Review of Medicine, 1963, 14, 349

Weatherall, D J, and Bradley, J, Blood and its Disorders, p 1387. Oxford, Blackwell, 1974

Edinburgh, Churchill Livingstone, 1977.

Is there a time factor in renal allograft rejection?

SIR, - The claim by Dr M S Knapp and others (13 January, $p$ 75) to be able to accurately time rejection episodes in renal allograft recipients has interested us. We have therefore analysed 21 rejection episodes in 21 patients in the first postoperative month by extrapolating sequential measurements of plasma creatinine as described. ${ }^{1}$ All blood samples were taken at approximately 0900 . The rejection episodes were all well documented by abrupt changes in plasma creatinine concentration and in all cases there was additional clinical evidence. In many cases there was also histological evidence. We have found, contrary to Dr Knapp and his colleagues, a random distribution of rejection episodes throughout the day and night, with 11 episodes occurring between 2300 and 1100 and 10 episodes between 1100 and 2300 .

We have recently, for other reasons, changed the time of administration of immunosuppressive agents in our renal allograft recipients

from 2000 to 0800 . In the absence of any other changes in therapy, we have therefore been able to compare the influence of dose timing on allograft function. The number of rejection episodes and the severity of rejection as assessed by the total dose of methylprednisolone (given as bolus doses during acute rejection episodes) in the first six weeks after transplantation were analysed. Eight patients who received immunosuppressives at 2000 were matched as closely as possible with respect to age, sex, and degree of HLA matching with the same number of patients receiving immunosuppressives at 0800 . All patients studied were transplanted within the same 12-month period.

It was clearly seen that there were no significant differences between the two groups with respect to age, sex, HLA matching, number of rejection episodes, total dose of prednisolone, and graft function at the end of six weeks after transplantation. We have therefore found no evidence of a circadian rhythm in renal allograft rejection and no influence of the time of administration of immunosuppressive agents on the frequency and severity of rejection episodes during the first six postoperative weeks. The accuracy of timing rejection episodes by the method described in the paper is in our opinion questionable because of the errors inherent in extrapolation.

M K Chan
K Farrington

Department of Nephrology and Transplantation, London NW3

SIR,-Dr M S Knapp and others (13 January, p 75) have suggested that renal allograft rejection is a sudden event, basing their assertion on plots of the reciprocal of plasma creatinine concentration against time (since transplantation) for selected rejection episodes. These episodes were chosen because the values before and after rejection lay approximately on two straight lines, but they do not say how many rejection episodes do not look like this.

After transplantation (or after the kidney starts to function) the plasma creatinine falls from a very high concentration to a relatively stable low level. Rejection will be accompanied by a rise in creatinine concentration which may become very rapid. The fall after transplantation and the rise associated with rejection are two separate processes, and the effect described by Knapp et al will be apparent only if rejection starts before the creatinine level has stabilised. Otherwise the data will show a level plateau between the two periods of change (whether or not reciprocals are used). It would be interesting to know how many of their 22 rejection episodes occurred within a week of the kidney's starting to function or a previous rejection episode.

The method of fitting two straight lines and taking the extrapolated point of intersection as the time of rejection has several weaknesses. Firstly, extrapolation is a hazardous procedure. Secondly, there are problems of allocating points near to the intersection to the "up" or "down" phase. Thirdly, the points on the second line may well have been affected by the treatment (see their figure). Finally, the reciprocal transformation will produce values of unequal reliability-errors in low (raw) values will be magnified.

My figure shows that if the data in their 


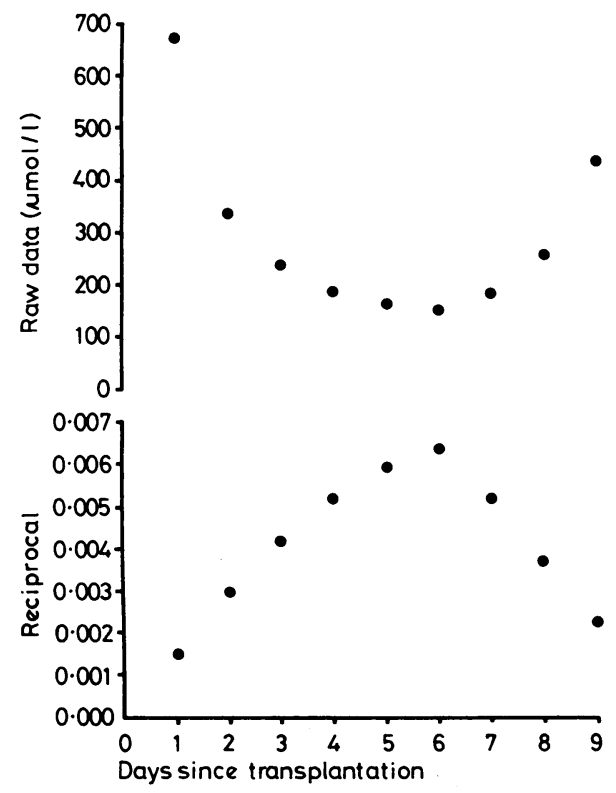

Serum creatinine concentration by time.

figure are transformed back into the original units serum creatinine concentrations change smoothly with time. A quadratic curve fits the data reasonably well, although a more appropriate model might be to fit separate exponential curves to the periods of improvement and deterioration. It is hard to see why they have chosen to use the artificial method of taking reciprocals rather than study the raw data. The authors rightly say that "a time when renal allograft rejection occurs can be determined only if there is a sudden event rather than slow changes from a non-rejecting to a rejecting state." I cannot accept that they have established this.

Lastly, their approach assumes that onset of rejection results in an immediate rise in serum creatinine. Is this really so ?

\section{G Altman}

Division of Computing and Statistics,

Clinical Research Centre,

Northwick Park
Harrow, Middx

***We sent a copy of this letter to the authors, whose reply is printed below.-ED, $B M \mathcal{H}$.

SIR,-Mr Altman does not understand why we plotted the reciprocal rather than the untransformed, "raw" data, but to use the raw data is to overlook the biology of plasma creatinine concentration measurements when used as a guide to renal function.

It is logical to use the reciprocal because the relation between plasma creatinine concentration and glomerular filtration rate is hyperbolic and curvilinear whether studied in vivo or as a mathematical model. ${ }^{1}$ It was a realisation of this that led physicians to plot sequential plasma creatinine measurements as the reciprocal, and so demonstrate the straight line relations, which can predict by extrapolation the subsequent course of some patients in renal failure. ${ }^{2-4}$ This suggested that in these patients nephron loss proceeded at a constant rate while in others, whose sequences of plasma creatinine measurements were best demonstrated on semilogarithmic plots, nephron loss proceeds at a rate which is a fixed percentage of the number remaining. In a disorder in which the loss of nephrons is sudden and total the subsequent increase in plasma creatinine should be demonstrated as a straight line against time if raw data are used, as there will be no renal loss but continued production at a constant rate (as it is assumed to be whenever creatinine is used as a guide to change in renal function)

The loss of function with allograft rejection might follow any of these alternative courses and our choice of the reciprocal is based on comparisons between the three types of plot. We are now comparing the alternatives more carefully, having collected more samples each day than in our reported examples, and are hoping to obtain more information about the pathophysiology of rejection and to confirm our initial conclusion that the reciprocal is the transformation that most frequently demonstrates the changes in a way that is analysable by linear regression, and avoids the need for the multiple data points required when considering curves.

We have, as stated in our.paper, subjected the data to analysis using a method developed by $\mathrm{Smith}^{\mathbf{5}}$ and obtained further evidence for our hypothesis. This method was designed to select the most likely time for a change to have occurred, and does not make an arbitrary allocation of points to a phase of improvement or deterioration. It calculates the probability of there having been a change at any time during the sequence of results considered. This programme also gives more emphasis to points close to the time of change than to those distant from it, thus reducing any "magnification of error" introduced by transforming low creatinine values to the reciprocal. The objective was to determine the time of onset of deterioration, in contrast with the continuation of improvement or stability, and we have other facts which support our thesis that the point where our "line of improvement" and "line of deterioration" intercept represent moments of crisis for renal function. We frequently noted a sudden increase in weight on the date of intercept (not a few days before, when $\mathrm{Mr}$ Altman's method suggests that a decline in renal function may have started). Also, when studying circadian variations in electrolyte excretion after transplantation we identified isolated days when the reversed or absent circadian variations in sodium excretion (observed after transplantation) were distorted by a transient nocturnal drop in sodium output and a subsequent daytime natriuresis. These abnormal days were observed to coincide with the intercept of the lines of improvement and lines of deterioration. ${ }^{6}$ This provides support for our thesis that there is a sudden event a this time causing an alteration in one mathematically predictable course and heralding a change to another. There is considerable support for this view from experimenta studies in renal transplantation, which show abrupt changes in renal perfusion associated with rejection.

Our method of using the reciprocal identifies the onset of rejection several days earlier than methods used by many authors, who often use a rise in "non-transformed" plasma creatinine of $25^{\circ}$ as their definition of rejection and do not use weight correction to remove the effects of water retention at the time of rejection. A "line of deterioration" has been observed to develop following periods of stable function, arguing against $\mathrm{Mr}$ Altman's suggestion that we have been observing the end of a sequential process-our "time of rejection" representing the minimum point in a continuous sequence of deterioration starting earlier than our "abrupt event."

Increased numbers of samples at the time when renal function changes are essential before our hypothesis can be fully proved. These are being collected in a prospective study.

\section{MARTIN S KNAPP} R PoWNALL

City Hospital, Nottingham

Doolan, P D, Alpen, E L, and Theil, G B, American Journal of Medicine, 1962, 32, 65.
${ }^{2}$ Mikh, W E, and Waker, M, Clinical Research, 1976,

24, 407.
${ }^{3}$ Rutherford, W E, et al, Kidney Intcrnational, 1977, 11,62 .

Knapp, M S, et al, Lancet, 1977, 2, 1183

Smith, A F M, and Cook, D G; submitted for

publication.
Kountz, S, Lamb, D R, and Cohn, R, fournal of the

American Medical Association, 1965, 191, 12. 794.

\section{Delayed haemorrhagic disease of the newborn}

SIR,-The report by Drs N A Cooper and M A Lynch (20 January, p 164) of delayed haemorrhagic disease of the newborn complicated by extradural haematoma underlines a rare but definite problam. Recent similar experience ${ }^{1}$ with a 6 -week-old breast-fed baby admitted with apparent "viral" infection who developed an extensive haematoma at the lumbar puncture site and prolonged bleeding after venepuncture raised similar anxieties. Since breast milk contains $15 \mu \mathrm{g} / \mathrm{l}$ of vitamin $\mathrm{K}$ compared with the $60 \mu \mathrm{g} / \mathrm{l}$ of cows' milk, breast-fed babies are at greater risk from such a bleeding diathesis and even severe intracerebral bleeding, as reported. To prevent even a very occasional case the routine and safe administration of vitamin $\mathrm{K}$ to at least all babies who are breast-fed seems logical. Certainly in such babies administration of vitamin $\mathrm{K}$ or at least estimation of prothrombin time might be prudent when admission to hospital and invasive procedures are necessitated by infection or malaise.

A Minford O B EDEN

Department of Child Life and Health, Royal Hospital for Sick Children,

Minford, A, and Eden, O B, Archives of Diseases of Childhood. In press.

SIR,-Drs N A Cooper and M A Lynch have reported subdural haemorrhage, occurring at 6 weeks in a baby born at 37 weeks' gestation, who had vitamin $\mathrm{K}$ deficiency associated with breast-feeding (20 January, $p$ 164). The incidence of significant bleeding from this cause after the neonatal period is unknown, but it is generally thought to be rare. We wish to record gastrointestinal bleeding due to vitamin $\mathrm{K}$ deficiency in a full-term, breastfed baby aged 4 weeks.

The infant, who weighed $3.3 \mathrm{~kg}$ at birth, had not received vitamin $\mathrm{K}$ prophylaxis. He was fully breast-fed and had thrived until the age of 4 weeks, when he developed epistaxis and melaena. On examination his general condition was good; he was mildly jaundiced and had two small recent bruises. His serum bilirubin concentration was $130 \mu \mathrm{mol} / 1(7.6 \mathrm{mg} / 100 \mathrm{ml})$; serum asparate aminotransferase and alanine aminotransferase concentrations were normal. There were no bilirubin, urobilinogen-reducing substance, or abnormal amino-acids in the urine. The haemoglobin concentration was $14.2 \mathrm{~g} / \mathrm{dl}$, white blood cells $13.1 \times 10^{9} / 1$, platelets normal, and serum fibrinogen $5.0 \mathrm{~g} / 1$ (normal); but other tests of clotting function were grossly abnormal, indicating simple vitamin $\mathrm{K}$ deficiency. The prothrombin time was corrected to 17 seconds in vitro with normal plasma $(50 / 50$ 